\title{
EXPERIENCIA EN METODOLOGÍAS ACTIVAS: PROYECTO PARA LAS ASIGNATURAS INTRODUCCIÓN A LA CONTABILIDAD Y CONTABILIDAD FINANCIERA EN EL GRADO DE RELACIONES LABORALES Y RECURSOS HUMANOS DE LA UPV/EHU
}

Experience in Active Methodologies: Project for Introduction to Accounting and Financial Accounting Subjects in the Grade of Labor Relations and Human Resources of the University of the Basque Country (UPV/EHU)

\author{
Ainhoa Saitua Iribar \\ $\mathrm{UPV} / \mathrm{EHU}$ \\ Orcid: 0000-0002-6937-1753 \\ ISABEL VÁzQUEz Arias \\ $\mathrm{UPV} / \mathrm{EHU}$ \\ Orcid: 0000-0003-2028-3813
}

\section{ABSTRACT}

Este articulo presenta los resultados de la experiencia en la implementación del Aprendizaje Basado en Proyecto (ABPy) para las asignaturas de Contabilidad en el grado de Relaciones Laborales y Recursos Humanos. Hemos podido comprobar que el alumnado puede aprender de forma alternativa a la tradicional. La formulación de una pregunta motriz como eje de un proyecto ayuda a visualizar la utilidad de cada 
una de las asignaturas en la vida real y, por lo tanto, entendemos que fomenta la motivación del alumnado. Sin embargo, desde el curso 2013/2014, en el que se produjo la primera implantación de la metodología de ABPy, hasta el curso 2016/2017, la tasa de rendimiento ha disminuido en todos los grupos. Así, creemos que por mucho esfuerzo pedagógico que realice el profesorado, es el alumnado quien finalmente determina con sus capacidades iniciales $y$, después, con su asistencia, actitud y esfuerzo continuado en el tiempo, el mayor o menor éxito en su proceso de aprendizaje y, finalmente, su nivel de éxito en la superación de las materias correspondientes.

Palabras clave: metodologias activas, Relaciones Laborales, Contabilidad, rendimiento académico.

Artikulu honek Lan Harreman eta Giza Baliabideetako graduan kontabilitateko irakasgaietan Proiektuan Oinarritutako Ikaskuntza (POI) metodoa ezartzearen esperientziaren emaitzak aurkezten ditu. Ikasleek modu tradizionalaren alternatiba baten bidez ikas dezaketela aurkitu dugu. Proiektua gidatzeko galdera-gako bat aurkezteak irakasgai bakoitzak bizitza errealean duen erabilgarritasuna ikustarazten laguntzen du eta, hortaz, ikasleen motibazioa bultzatzen laguntzen duela ulertzen dugu. Hala ere, POI metodologia ezartzearen lehen ikasturtea 2013/2014 geroztik eta 2016/2017 arte, etekin-tasak talde guztietan jaitsi dira. Horrela, irakasleek hezkuntza lanetan ahalegin asko eginda ere, uste dugu ikasleak direla, azken finean, beren hasierako gaitasunekin eta, ondoren, beren bertaratzearekin, jarrerarekin eta denboran zehar egindako ahalegin iraunkorrarekin, euren ikaskuntza prozesuan eta, ondorioz, ikasgaiak gainditzearen prozesuan, arrakasta handiagoa edo txikiagoa lortzea baldintzatzen dutenak.

Hitz gakoak: metodologia aktiboak, Lan Harremanak, Kontabilitatea; errendimendu akademikoa.

This article presents the results of the the implementation of Project Based Learning (PjBL) for the subjects of Accounting in the degree of Labor Relations and Human Resources. We have verified that students can learn in an alternative way to the traditional one. The formulation of a driving question as the axis of the project helps to visualize the usefulness of each one of the subjects in the real life, and therefore, we understand that it promotes the student's motivation. However, since the 2013/2014 course of first implementation of the PjBL methodology, until the 2016/2017 academic year, the rate of performance has decreased in all groups. Thus, we believe that although teachers make a lot of pedagogical effort, it is the students it is the students who finally determine with their initial abilities, and then with their assistance, attitude and continuous effort over time, the greater or less success in their learning process, and consequently, their level of success in overcoming the corresponding subjects.

Keywords: active methodologies, Labor relations, Accounting, academic performance. 


\section{SUMARIO}

Sumario. 1. Introducción. 2. Formación sobre Aprendizaje Basado en Proyecto (ABPy) en el marco del programa ERAGIN. 3. Diseño del proyecto para las asignaturas Introducción a la Contabilidad y Contabilidad Financiera. 3.1. Contexto de las asignaturas y formulación general del proyecto. 3.2. Metodología y evaluación. 4. Implantación en el aula, seguimiento y resultados del proyecto. 4.1. Puesta en marcha. 4.2. Seguimiento y resultados de la primera implementación. 4.3. Evolución posterior de los resultados académicos, implicaciones y nuevas tareas. 5. Conclusiones y reflexiones finales.

\section{Introducción}

En el siglo xx se fueron produciendo importantes cambios en los entornos económicos, sociales y culturales que dibujan el tránsito de una sociedad industrial a la de la información, el conocimiento y/o el aprendizaje; en definitiva, una sociedad en la que la capacidad individual y colectiva para conocer y para aprender es el elemento sustancial (Domínguez, 2009). Pero en el siglo xxi los modos de producción y transmisión del conocimiento se están viendo sensiblemente modificados debido, en gran medida, a la expansión de las nuevas tecnologías y muy especialmente de internet, de forma que en la actualidad es posible el acceso en tiempo real a gran cantidad de información inabarcable por la mente humana, de modo que en este parámetro de fuerte transformación, resulta necesario preguntarse en qué dirección hay que dirigir el cambio en la formación superior, para qué, por qué y cómo materializarlo en la práctica. Las universidades, como instituciones responsables de la producción y transmisión de conocimiento científico, tratan de adaptarse resultando en cambios que afectan a la vida de las aulas y al quehacer docente. En el caso de las universidades europeas el primer detonante del cambio vino de la mano del Espacio Europeo de Educación Superior (EEES), lo que ha acentuado la presión por la mejora de la calidad de la enseñanza universitaria y uno de cuyos cambios más tangibles consistió en la introducción del ECTS (European Credit Transfer System), que mide el trabajo realizado por la o el estudiante dentro del sistema (Fernández y Alkorta, 2014: 14).

Siguiendo la exposición de motivos que realiza la Universidad del País Vasco/Euskal Herriko Unibertsitatea en su página web (UPV/EHU, 2011), en 
la actualidad el profesorado universitario ya se ha familiarizado con términos como competencias, evaluación continua, modalidades docentes, etc., pero no puede conformarse con considerar la educación universitaria como un proceso de transmisión de la información del profesorado al alumnado, sino que debe proporcionar oportunidades para un aprendizaje significativo y crítico, que sirva para construir nuevo conocimiento. En concreto, la UPV/EHU ha desarrollado una reflexión sobre cómo afrontar estos retos en el marco del modelo educativo denominado IKD, que establece una interacción entre diferentes niveles co-responsables de su materialización: i) desarrollo curricular, ii) desarrollo social y territorial, iii) desarrollo profesional, iv) desarrollo institucional, y v) educación activa. Este modelo aspira a formar personas conscientes y sensibles con lo que ocurre en su entorno, que sean capaces de adaptarse a los cambios continuos y que puedan responder de forma creativa y ética a problemas nuevos.

En lo que respecta a los métodos de enseñanza-aprendizaje, las denominadas metodologías activas se han desarrollado en numerosas universidades europeas e internacionales, que las han utilizado con evidencias de buenos resultados porque contribuyen a enfrentar el reto de formar en competencias (UPV/EHU, 2011). Entre las ventajas de utilizarlas estarían: la mayor motivación, interés e implicación del alumnado; menor tiempo de finalización de los estudios y menor tasa de abandono; mayor retención de los conocimientos adquiridos; mayor desarrollo de habilidades y competencias profesionales; mayor conexión entre la teoría y la aplicación, entre el conocimiento previo y el que se va aprendiendo; y mayor integración de los conocimientos entre diferentes disciplinas.

En general, estas metodologías activas comparten algunos aspectos que las caracterizan: el punto de partida del proceso de aprendizaje es un problema, proyecto o caso. Durante el mismo se pone el énfasis en la formulación de preguntas, en lugar de mostrar directamente las respuestas. Así, son los/as estudiantes quienes deben deducir los objetivos de aprendizaje y los contenidos implicados para resolver el problema, caso, o desarrollar el proyecto. El problema es el contexto en el que se relacionan o integran los contenidos necesarios para resolverlo. La metodología implica una reducción sustancial de las exposiciones docentes en el aula porque los/as estudiantes toman parte activa en la búsqueda de información para aprender los contenidos implicados, sin que tengan que ser expuestos en el aula por el profesor/a. Y es en la relación entre práctica y teoría donde los/as estudiantes aprenden a relacionar la experiencia concreta con la teoría. No se imparte en primer lugar la teoría para luego ponerla en práctica en la resolución de algún ejercicio, sino que se analiza en primer lugar un problema para deducir los objetivos de aprendizaje que, luego, son trabajados por el grupo de estudiantes con la guía del profesorado, favoreciendo el aprendizaje auto dirigido. La mayoría de los procesos de aprendizaje en estas metodologías tiene lugar en grupos de estudiantes, trabajando cooperativamente, y en interacción continua con el/la docente. Los/as estudiantes reciben retroalimentación continua e inmediata du- 
rante el proceso de aprendizaje, lo cual conlleva la utilización de un sistema de evaluación continuada que valore el progreso en el desarrollo de competencias, y permita reorientar y adaptar la planificación docente a las necesidades que van surgiendo. De este modo, implementar una enseñanza basada en metodologías activas supone algo más que ser un/a especialista en una rama de conocimiento científico: supone desarrollar competencias docentes específicas que se orientan a conseguir que el/a estudiante participe activamente en su aprendizaje.

Con estos antecedentes, el objetivo de este artículo consiste en presentar los resultados de la experiencia en la primera implementación del Aprendizaje Basado en Proyecto (ABPy) para las asignaturas de Introducción a la Contabilidad y Contabilidad Financiera en el grado de Relaciones Laborales y Recursos Humanos, así como su evolución, analizando las tasas de rendimiento académico de un periodo total de 5 años a partir del curso anterior a la primera implantación del ABPy. Tras esta introducción, el apartado dos explica cómo fue el proceso de diseño del proyecto para las dos asignaturas a partir de la formación recibida en el programa ERAGIN de la UPV/EHU, el tercero explica cómo se diseńó el proyecto, el cuarto da cuenta de la primera implementación en el curso 2013/2014 y de los resultados obtenidos en los ańos posteriores. Por último, el apartado quinto se dedica a las reflexiones finales.

\section{Formación sobre Aprendizaje Basado en Proyectos (ABPy) en el marco del programa ERAGIN}

En 2010, por iniciativa del Vicerrectorado de Estudios de Grado e Innovación de la UPV/EHU y bajo la dirección y supervisión del Servicio de Asesoramiento Educativo (SAE), se propuso el programa ERAGIN para asesorar en el diseño, desarrollo y evaluación de una enseñanza basada en metodologías activas, en particular, en estrategias fundamentadas en el Aprendizaje Basado en Problemas (ABP), en el Método del Caso (MdC) y en el Aprendizaje Basado en Proyectos (ABPy), junto con el aprendizaje cooperativo (AP). El programa pretendía profundizar en la utilización sistemática y coherente de las metodologías mencionadas, incorporando a la experiencia previa de las personas docentes nuevas estrategias y técnicas de enseñanza que faciliten el aprendizaje más inductivo y más cercano a la resolución de situaciones profesionales reales de carácter abierto (UPV/EHU, 2011). El programa, del que se han llevado a cabo 5 ediciones, tenía carácter voluntario mediante convocatoria anual para todo el profesorado, si bien tenía un número limitado de plazas (75), debido a la disponibilidad de recursos humanos.

ERAGIN se planteó con los siguientes objetivos generales: 1) formar profesores y profesoras de la UPV/EHU en metodologías activas de enseñanza para 
que, en un futuro, fueran referente en la práctica de excelencia de estas estrategias y pudieran formar y asesorar a otro profesorado de su centro y/o área de conocimiento; 2) promover durante la formación una actitud reflexiva ante la enseńanza de la asignatura, el desarrollo de competencias basadas en la innovación y la evolución de procesos y resultados; 3) poner en marcha un centro de recursos digitales que se convirtiera en un referente de la documentación y la divulgación de nuevas experiencias en la enseñanza con las tres metodología mencionadas. En concreto, el programa de formación tenía como objetivo mediar las concepciones que tenía el profesorado sobre su actividad docente y modificar su práctica hacia metodologías activas de enseñanza. En este caso, se decidió recurrir a la mentoría de larga duración como forma de acompañar al profesorado tanto en el diseño de una propuesta activa como en su implementación en el aula (Guisasola y Garmendia, 2014: 32).

Entre las posibles modalidades, debido al carácter progresivo de los conocimientos que se trabajan en estas asignaturas de contabilidad en las que se reproduce el ciclo real de la contabilidad a elaborar durante el ejercicio económico de cualquier empresa, nuestra opción fue la de Aprendizaje Basado en Proyectos (APBy). En este caso, el proceso de aprendizaje se basa en el desarrollo de un proyecto que establece una meta determinada como producto final (Solomon, 2003) y cuya metodología está relacionada con el trabajo en equipo (Cullen et al., 2004; Carrasco et al., 2009). Las características más importantes de la metodología ABPy son las siguientes (Aliane, 2006: 2):

1. El ABPy se desarrolla en un entorno real y experimental. Esto ayuda al alumnado a relacionar los contenidos teóricos con el mundo real, lo cual recae en una mejor receptividad para aprender los conceptos teóricos.

2. El alumnado toma un papel activo en el proyecto, ya que tiene que marcar el ritmo y la profundidad del aprendizaje.

3. Es una metodología que motiva al alumno/a por lo que puede constituir una herramienta para la mejora del rendimiento académico.

4. Permite desarrollar competencias específicas de la asignatura pero también competencias generales, como el trabajo en equipo.

5. El rol de la persona docente no es el de un/a observador/a pasivo/a. Toma el rol de un/a tutor/a y consultor/a. Tiene que suministrar los conocimientos necesarios en los momentos adecuados para estimular el proceso de aprendizaje.

Las fases del programa ERAGIN se desarrollaron como estaba previsto. En primer lugar, las profesoras, que ya habíamos trabajado coordinadamente tanto en la docencia de las asignaturas que impartimos como en la reflexión sobre las innovaciones que está suponiendo la adaptación a los nuevos grados (Saitua y Vázquez, 2007a y 2007b), recibimos la formación en talleres de iniciación en 
metodologías activas de enseñanza, con una duración de 20 horas repartidas a lo largo de 3 días en una semana. El propio taller era un modelo de ejemplo en la utilización de la metodología. Seguía una secuencia de actividades que favorecían la reflexión y la discusión grupal. Como participantes experimentábamos la metodología en las actividades diseñadas asumiendo el rol de estudiantes para a continuación analizar, debatir las estrategias desde el punto de vista docente. En las tres primeras ediciones los talleres fueron impartidos por personas externas a la UPV/EHU, pero en la cuarta edición, en la que participamos, se realizó mediante recursos propios. Después, a lo largo de 4 meses, realizamos el diseño de la asignatura, con apoyo de una mentoría colaborativa, para integrar la metodología activa e investigación del caso, problema o proyecto en una propuesta que abarcara al menos el $25 \%$ de la asignatura elegida y para la que se debían entregar diversos documentos. La persona mentora debía tener experiencia previa en la metodología y su papel no era el de obligar, sino el de hacernos reflexionar sobre estrategias de enseñanza alternativas y sus implicaciones en la práctica educativa. En el curso siguiente tuvo lugar la implementación de la propuesta en el aula y validación externa de la propuesta metodológica, también con acompañamiento de mentoría colaborativa que incluía, al menos, una observación directa en el aula. Por último, tuvo lugar la evaluación de resultados y divulgación. Una vez que la propuesta fue evaluada como positiva, fue objeto de publicación en el correspondiente repositorio de la UPV/ EHU (Saitua y Vázquez, 2014).

\section{Diseño del proyecto para las asignaturas Introducción a la Contabilidad y Contabilidad Financiera}

Tras la fase de formación en los talleres presenciales en febrero de 2013, el diseño del proyecto tuvo lugar durante los meses de marzo a junio de ese mismo año.

\subsection{Contexto de las asignaturas y formulación general del proyecto}

Las asignaturas en las que propusimos implementar el programa ERAGIN fueron Introducción a la Contabilidad (IC) y Contabilidad Financiera (CF), que se imparten en 2..$^{\circ}$ curso del Grado del Relaciones Laborales y Recursos humanos, en el $1 .^{\circ}$ y $2 .^{\circ}$ cuatrimestre respectivamente. Son las únicas asignaturas obligatorias del área contable en esta titulación y cuentan con 6 créditos presenciales (4 horas de clase presencial por semana) cada una. En ambas asignaturas se imparten 3 grupos docentes ( 1 en euskera por la mańana y dos de castellano: uno de mañana y otro de tarde). El número total, aproximado, de personas matriculadas en cada materia es de 125 . 


\subsubsection{Pregunta motriz y escenario del proyecto}

El objetivo que se plantea es que parte del proceso de enseńanza aprendizaje se adquiera mediante un proyecto con una pregunta motriz a la que habrá que dar respuesta al finalizar la segunda asignaturas: «La empresa de la que llevamos la contabilidad ¿̇repartirá dividendos al cierre del ejercicio?». Por lo tanto, se trata de un único proyecto dividido en dos fases, con dos escenarios que se corresponden con las dos asignaturas.

-Introducción a la Contabilidad (IC). En la asesoría laboral-fiscal-contable en la que desempeńáis vuestra labor de experto/a en Derecho Laboral, el/ la contable ha caído de baja, por lo que debéis haceros cargo de la contabilidad de un cliente (una empresa comercial), desde el comienzo del periodo 20X1. Como nunca habéis llevado la contabilidad de este cliente, tenéis que iniciar el proyecto buscando los documentos de partida del ejercicio 20X0 en el Registro Mercantil de la provincia donde tenga su domicilio social. Además, habréis de contabilizar las operaciones normales del ciclo de explotación (compras y ventas de existencias con descuentos y anticipos, pago de nóminas, etc.).

- Contabilidad Financiera (CF). Una vez que el ciclo de explotación estaba en marcha, la empresa también ha realizado operaciones de inversión en inmovilizado técnico e inversiones financieras, así como operaciones de financiación y creación de provisiones de pasivo, etc. Finalmente, se decide sobre la propuesta correspondiente a la aplicación del resultado neto de impuestos.

\subsubsection{Competencias, temario y resultados de aprendizaje}

Para poder definir las tareas y su evaluación, en primer lugar es necesario establecer las competencias y los objetivos de aprendizaje. Así, las guías docentes de las asignaturas IC y CF, adaptadas a los criterios de la memoria de la ANECA y publicadas en la página web del centro, especifica, las competencias, resultados de aprendizaje, temario, etc.

En cuanto a las competencias a desarrollar, el equipo de coordinación del Grado en su día realizó un esfuerzo para elaborar la guía docente de las competencias transversales y las específicas de la titulación. Así, en un principio, se quedó que, al menos, cada asignatura valore una competencia transversal (aunque pueda también trabajar otras), y a las asignaturas IC y CF les corresponde valorar la competencia «Adaptación al entorno». De modo que a lo largo del proyecto se desarrollarán las siguientes competencias: 
Tabla 1 Competencias de las asignaturas Introducción a la Contabilidad
y Contabilidad Financiera

\begin{tabular}{|c|c|}
\hline Introducción a la Contabilidad (IC) & Contabilidad Financiera (CF) \\
\hline \multicolumn{2}{|c|}{ COMPETENCIAS TRANSVERSALES (CT) } \\
\hline \multicolumn{2}{|c|}{$\begin{array}{l}\text { CT: Adaptación al entorno: Afrontar los cambios técnicos, sociales, laborales y culturales de la sociedad a } \\
\text { través de un proceso de formación continuado a lo largo de su vida. }\end{array}$} \\
\hline \multicolumn{2}{|c|}{ COMPETENCIAS ESPECÍFICAS DE GRADO (CG) } \\
\hline \multicolumn{2}{|c|}{ CG: Gestionar y asesorar de modo eficaz en cuestiones relativas a fiscalidad y contabilidad. } \\
\hline \multicolumn{2}{|c|}{ COMPETENCIAS ESPECÍFICAS DE ASIGNATURA (CA) } \\
\hline $\begin{array}{l}\text { IC_CA1. Comprender activamente, esquematizar, } \\
\text { memorizar y utilizar información sobre el sis- } \\
\text { tema de información contable, su metodología y } \\
\text { sus normas básicas. } \\
\text { IC_CA2. Recoger información contable de una em- } \\
\text { presa real, para elaborar un informe e interpretar } \\
\text { de forma oral o escrita su situación patrimonial } \\
\text { y su resultado. } \\
\text { IC_CA3. Registrar contablemente y explicar de } \\
\text { forma oral o escrita las operaciones básicas del } \\
\text { ciclo de explotación de una pequeńa empresa, } \\
\text { utilizando el Libro Mayor y el Libro Diario para } \\
\text { realizar el seguimiento del valor del patrimo- } \\
\text { nio y sus componentes, tanto mediante trabajo } \\
\text { cooperativo como individual. } \\
\text { IC_CA4. Elaborar el Balance de Situación y la } \\
\text { cuenta de Pérdidas y Ganancias de una empresa } \\
\text { según el orden y formato establecidos, para re- } \\
\text { presentar su patrimonio y su resultado, tanto } \\
\text { mediante trabajo cooperativo como individual. }\end{array}$ & $\begin{array}{l}\text { CF_CA1. Comprender la necesidad de la planifi- } \\
\text { cación y normalización contable para aumen- } \\
\text { tar la eficacia de la información financiera, de } \\
\text { forma que pueda satisfacer las necesidades de } \\
\text { los usuarios en la toma de decisiones, y en- } \\
\text { tender y aplicar el PGC como máxima expre- } \\
\text { sión de la normalización contable en España. } \\
\text { CF_CA2. Conocer los aspectos de la regulación } \\
\text { contable referidos a los plazos relativos a la } \\
\text { elaboración, auditoría, aprobación y depósito } \\
\text { de los Estados Contables que se publican, } \\
\text { para que cualquier usuario pueda acceder a la } \\
\text { información contable. } \\
\text { CF_CA3. Aplicar las normas de contabilidad vi- } \\
\text { gentes específicas para valorar y registrar cier- } \\
\text { tas operaciones de inversión, financiación y } \\
\text { explotación de las empresas, así como identi- } \\
\text { ficar su efecto en el patrimonio y en el resul- } \\
\text { tado de la empresa. } \\
\text { CF_CA4. Elaborar el Balance de Situación y la } \\
\text { cuenta de Pérdidas y Ganancias de una em- } \\
\text { presa según el orden y formato estableci- } \\
\text { dos, para representar su patrimonio y su re- } \\
\text { sultado, tanto mediante trabajo cooperativo } \\
\text { como individual. } \\
\text { CF_CA5. Interpretar y explicar, tanto de forma } \\
\text { individual como cooperativa, las operaciones } \\
\text { registradas en base a los principios y normas } \\
\text { de contabilidad, identificando la sustancia } \\
\text { del hecho económico que se refleja, para que, } \\
\text { ante posibles modificaciones de normas en el } \\
\text { futuro, pueda adaptar todo el proceso conta- } \\
\text { ble (valoración, registro, presentación) a esa } \\
\text { nueva normativa. }\end{array}$ \\
\hline
\end{tabular}

Fuente: Elaboración propia. 
A lo largo de este proyecto se desarrollan los siguientes 15 temas.

Tabla 2

Temario de las asignaturas Introducción a la Contabilidad y Contabilidad Financiera

\begin{tabular}{l}
\hline Introducción a la Contabilidad (IC) \\
\hline Tema 1. La empresa y la contabilidad \\
1.1. La actividad económica y la empresa. \\
1.2. El sistema de información contable. \\
Tema 2. El balance: el estado de la información \\
empresarial
\end{tabular}

2.1. El Patrimonio.

2.2. El Estado de la posición económico-financiera. El Balance de Situación.

2.3. Cambios patrimoniales.

Tema 3. El resultado: el indicador de la eficiencia empresarial

3.1. Concepto de Resultado.

3.2. El reconocimiento del gasto.

3.3. Transacciones que generan ingreso.

3.4. El modelo de representación del Resultado: la Cuenta de Pérdidas y Ganancias.

Tema 4. El método contable

4.1. Introducción.

4.2. La cuenta como instrumento de representación y sus tecnicismos.

4.3. Funcionamiento de las cuentas de acuerdo con la partida doble.

4.4. Registro de los hechos contables.

Tema 5. El ciclo contable

5.1. Introducción.

5.2. Orden de las operaciones en el Libro Diario y Libro Mayor.

5.3. El Balance de Comprobación de Sumas y Saldos.

5.4. La elaboración de las Cuentas Anuales.
Contabilidad Financiera (CF)

Tema 1. Comunicación de la información contable

1.1. Introducción. La información contable disponible según la legislación actual.

1.2. La información sobre Responsabilidad Social Corporativa y el uso no sexista del lenguaje.

Tema 2. Marco conceptual

2.1 La Regulación Contable.

2.2 El Marco Conceptual.

2.3 Inventario de Principios Contables.

Tema 3. El inmovilizado afecto a la explotación

3.1. El activo no corriente o inmovilizado: definición y presentación.

3.2. Inmovilizado Material: Definición y valoración.

3.3. Valoración posterior: Correcciones valorativas.

3.4. Inmovilizado Material en curso.

3.5. Operaciones posteriores a la puesta en condiciones de funcionamiento.

3.6. Ayudas a la inversión: Subvenciones para la adquisición de inmovilizado.

3.7. El Inmovilizado Intangible.

Tema 4. Las inversiones financieras

4.1. Las inversiones financieras y sus clases.

4.2. Inversiones financieras temporales y su cuadro contable.

4.3. Valores negociables.

4.4. Créditos no comerciales. 
EXPERIENCIA EN METODOLOGÍAS ACTIVAS: PROYECTO PARA LAS ASIGNATURAS INTRODUCCIÓN A LA CONTABILIDAD y Contabilidad Financiera en el grado de Relaciones Laborales y Recursos Humanos de La UPV/EHU

Introducción a la Contabilidad (IC)
Tema 6. La normalización contable
6.1. El fenómeno de la normalización contable.
6.2. Efecto de las normas internacionales en Es-
paña.

6.3. La regulación contable española.

6.4. El Plan General de Contabilidad.

\section{Tema 7. El tratamiento contable de almacén}

7.1. Introducción: El ciclo de explotación.

7.2. Clases de existencias.

7.3. Tratamiento contable de las existencias.

7.4. Tratamiento del IVA.

Tema 8. Personas y entidades deudoras y acreedoras por operaciones del tráfico

8.1. Operaciones del tráfico versus operaciones financieras.

8.2. Anticipos a personas y entidades proveedoras y de clientas.

8.3. Tratamiento contable del riesgo de cobro.

8.4. Los efectos comerciales.

8.5. Otras formas de gestión de créditos: el factoring.

Tema 9. Los recursos humanos

9.1. La nómina.

9.2. Otros gastos sociales.
Contabilidad Financiera (CF)

Tema 5. Los fondos propios

5.1. Introducción a los Fondos propios.

5.2. Cálculo del resultado neto del impuesto sobre beneficios.

5.3. Aplicación del resultado del ejercicio.

Tema 6. El pasivo

6.1. Las fuentes de financiación ajenas.

6.2. Valoración de los pasivos.

6.3. Provisiones: concepto y valoración.

Fuente: Elaboración propia. 
Y los resultados de aprendizaje que se esperan obtener son los siguientes:

Tabla 3

Resultados de aprendizaje de las asignaturas Introducción a la Contabilidad y Contabilidad Financiera

\begin{tabular}{|c|c|}
\hline Introducción a la Contabilidad (IC) & Contabilidad Financiera (CF) \\
\hline $\begin{array}{l}\text { IC_R1. Identifica los conceptos básicos } \\
\text { del sistema contable, así como del pa- } \\
\text { trimonio y del resultado en las cuentas } \\
\text { de una empresa (CA2). } \\
\text { IC_R2. Maneja los instrumentos princi- } \\
\text { pales del sistema contable: Libro dia- } \\
\text { rio y libro mayor (CA1; CA3). } \\
\text { IC_R3. Contabiliza, de acuerdo a las nor- } \\
\text { mas de registro y valoración corres- } \\
\text { pondientes, las operaciones básicas re- } \\
\text { lacionadas con existencias, riesgo de } \\
\text { cobro, IVA, anticipos, efectos a cobrar } \\
\text { y gastos de personal, así como las ope- } \\
\text { raciones de cierre de ejercicio, tanto en } \\
\text { el Libro diario como en el libro mayor } \\
\text { (CA1; CA3) (CT). } \\
\text { IC_R4. Elabora los documentos de sínte- } \\
\text { sis de la información contable: el Ba- } \\
\text { lance de Situación, y la Cuentas de } \\
\text { Pérdidas y Ganancias, de acuerdo a los } \\
\text { modelos oficiales (CA1; CA3; CA4) } \\
\text { (CT). } \\
\text { IC_R5. Interpreta, de forma oral o es- } \\
\text { crita, tanto las operaciones contabili- } \\
\text { zadas como los distintos documentos } \\
\text { de síntesis (CA3, CA5) (CT). }\end{array}$ & $\begin{array}{l}\text { CF_R1. Enuncia y explica cuáles son los } \\
\text { documentos disponibles en el Registro } \\
\text { Mercantil, a disposición de cualquier } \\
\text { interesado en la sociedad, una vez que } \\
\text { se hayan cumplido los plazos legales } \\
\text { de formulación, verificación, aproba- } \\
\text { ción y depósito (CA1; CA2) (CT9). } \\
\text { CF_R2. Identifica el criterio de recono- } \\
\text { cimiento y valoración específico para } \\
\text { cada elemento patrimonial, según las } \\
\text { circunstancias de la operación, den- } \\
\text { tro de las alternativas que proponga } \\
\text { la norma contable correspondiente } \\
\text { (CA3) (CT5). } \\
\text { CF_R3. Calcula el valor por el que se re- } \\
\text { conocerá el cargo o abono en la cuenta } \\
\text { patrimonial correspondiente (ya sea el } \\
\text { coste histórico o precio de adquisi- } \\
\text { ción, el coste amortizado, la amorti- } \\
\text { zación devengada en el ejercicio, etc.) } \\
\text { (CA3) (CT5). } \\
\text { CF_R4. Contabiliza la operación de } \\
\text { acuerdo con la normativa vigente y } \\
\text { muestra su efecto final en el Balance y } \\
\text { la Cuenta de Resultados (CA3; CA4) } \\
\text { (CT5). } \\
\text { CF_R5. Interpreta de forma oral o escrita, } \\
\text { tanto las operaciones contables como } \\
\text { los diferentes documentos de síntesis } \\
\text { (CA3; CA5) (CT5). }\end{array}$ \\
\hline
\end{tabular}

Fuente: Elaboración propia.

En la asignatura IC, que consta de 9 temas, propusimos trabajar mediante esta nueva metodología (en principio, sin que la profesora expusiera nada en el aula) únicamente los temas 2, 3, 7 y 8; y en la asignatura $\mathrm{CF}$, los temas 3, 4 y 5. En todo caso, el proyecto se plantea desde el inicio, y todos los temas están, de alguna manera, relacionados con él. 


\subsubsection{Tamaño de los grupos y criterios para formarlos}

Para el desarrollo del proyecto, los equipos/grupos de trabajo son de 3 personas. Los y las estudiantes son libres para formar los grupos y únicamente intervendrá la profesora cuando el/a alumno/a no sea capaz de introducirse en algún grupo en situaciones especiales. Los equipos/grupos de trabajo se conforman desde la segunda semana de clase, ya que el proyecto se plantea desde el inicio. Otras actividades de trabajo cooperativo en el aula, que no tengan relación con el proyecto, se desarrollarán en grupos formados al azar.

\subsubsection{Carga de trabajo del proyecto}

Cada asignatura tiene una carga de trabajo de 6 créditos, lo que supone 150 horas de trabajo para cada alumno/a, de las que 60 horas son presenciales y 90 no presenciales (tanto individuales, como grupales). Por lo tanto, las dos asignaturas suman 300 horas de trabajo por cada alumno/a. En cada asignatura, la carga estimada dedicada al proyecto son 2,4 créditos ( $40 \%$ de la asignatura), lo que supone alrededor de 60 horas de trabajo para el o la estudiante: 24 horas son de trabajo en el aula y 36 horas no presenciales, tanto de trabajo individual como de trabajo cooperativo. Por lo tanto, el proyecto global (teniendo en cuenta las dos asignaturas) suma un total de 120 horas para cada estudiante (48 presenciales y 72 no presenciales), repartidas en dos cuatrimestres del $2 .^{\circ}$ curso. Siendo los equipos/grupos de 3 personas, el proyecto conjunto requiere una dedicación total de 360 horas por cada equipo de trabajo.

\subsection{Metodología y evaluación}

El desarrollo de las competencias relacionadas con las dos asignaturas del proyecto requiere la búsqueda, análisis, síntesis y aplicación de información a través de diversos tipos de actividades. Para comprobar que se alcanzan los objetivos de aprendizaje, los y las estudiantes realizarán mapas conceptuales y resúmenes sobre algunos conceptos, registrarán operaciones en los libros contables y elaborarán los documentos de síntesis que informan sobre el patrimonio y el resultado, todo ello de forma presencial o bien fuera del aula, en ocasiones en grupo, y otras mediante trabajo individual. Se planifican actividades cooperativas desde el comienzo a fin de que las y los estudiantes se acostumbren a esta metodología y se impliquen en su proceso de aprendizaje.

\subsubsection{Entregables}

La realización de una actividad de aprendizaje en grupos de trabajo sitúa directamente al alumno/a en el centro del escenario, dejando a un lado al profesor/a que, únicamente, acompańa y guía al alumno/a en este proceso (Casasola et al., 2012). En nuestro caso, el alumnado debe presentar una serie de entregables: los entregables de aula (EA) están relacionados con la asignatura en 
su conjunto y el resto son entregables de proyecto (EP). Todos los entregables de proyecto se realizan en grupo. Cada grupo está obligado a trabajar de forma colaborativa, en el sentido de que todos los y las integrantes deben intervenir en todas y cada una de las tareas programadas en el proyecto.

En las actividades presenciales que realizamos en el aula, se aplican metodologías colaborativas que consisten en que cada miembro del grupo prepara una parte del tema y, después de explicar su parte al resto de miembros del grupo, completan colaborativamente la actividad correspondiente. Dicha actividad es corregida y evaluada, en la mayoría de los casos, por sus compañeros/as de otro grupo. Por ejemplo, los entregables de la asignatura IC son:

Tabla 4

Entregables en la asignatura Introducción a la Contabilidad

\begin{tabular}{|c|c|}
\hline Entregables de asignatura (EA) & Entregables de proyecto (EP) \\
\hline $\begin{array}{l}\text { IC_EA 1. Elaboración de un } \\
\text { mapa conceptual del tema } \\
1 \text { (grupal). } \\
\text { IC_EA 2. Resolución de ejerci- } \\
\text { cios: Elaboración de un Ba- } \\
\text { lance de Situación (grupal). } \\
\text { IC_EA 3. Resolución de ejerci- } \\
\text { cios: Elaborar la Cuenta de } \\
\text { Pérdidas y ganancias (gru- } \\
\text { pal). } \\
\text { IC_EA 4. Control individual } \\
\text { de contenidos mínimos (te- } \\
\text { mas 1-5). } \\
\text { IC_EA 5. Control individual } \\
\text { de contenidos mínimos (te- } \\
\text { mas 6-9) y repesca del pri- } \\
\text { mero. }\end{array}$ & $\begin{array}{l}\text { IC_EP1. Informe sobre la constitución del grupo, reflexión } \\
\text { sobre posibles disfuncionalidades, y normas de funciona- } \\
\text { miento consensuadas (grupal). } \\
\text { IC_EP 2. Presentación del escenario del proyecto. Reflexión y } \\
\text { puesta en común para el desarrollo del proyecto (grupal). } \\
\text { IC_EP 3. Recogida de información de unas cuentas anuales } \\
\text { reales (los datos que se desprenden de estas cuentas consti- } \\
\text { tuirán la situación de partida del proyecto). Copia del Ba- } \\
\text { lance y Cuenta de Resultados (grupal). } \\
\text { IC_EP 4. Interpretación preliminar sobre el estado de la situa- } \\
\text { ción: actividad de la empresa, el periodo sobre el que se in- } \\
\text { forma, la situación patrimonial y el resultado (grupal). } \\
\text { IC_EP 5. Esquemas conceptuales tipo poster sobre las opera- } \\
\text { ciones de existencias (grupal). } \\
\text { IC_EP6. Esquemas conceptuales tipo poster sobre las ope- } \\
\text { raciones de riesgo de cobro, efectos, anticipos y nóminas } \\
\text { (grupal). } \\
\text { IC_EP } 7 \text {. Relación de las operaciones a realizar por la empresa } \\
\text { en el ejercicio siguiente (grupal). } \\
\text { IC_EP 8. Informe del proyecto que incluirá tanto el análisis de } \\
\text { los datos de la empresa real y de las operaciones propuestas } \\
\text { en el ejercicio y su contabilización, como la elaboración de } \\
\text { las cuentas anuales al cierre del ejercicio (grupal). } \\
\text { IC_EA 9. Encuesta individual sobre el desarrollo del trabajo } \\
\text { en equipo y coevaluación de las aportaciones del resto de } \\
\text { miembros del equipo/grupo de trabajo (individual) } \\
\text { IC_EP 10. Exposición oral del proyecto (grupal). }\end{array}$ \\
\hline
\end{tabular}

Fuente: Elaboración propia. 


\subsubsection{Sistema de Evaluación}

La evaluación no debe basarse únicamente en calificar al alumnado, sino que debe ser, ante todo, una práctica reflexiva propia del/a docente, un control de calidad sobre lo que se hace, para después tomar decisiones que faciliten la mejora del aprendizaje (Gessa, 2011). De ahí que en los últimos años se hable de "evaluación orientada al aprendizaje» (Learning Oriented Assesment), que se apoya en tres aspectos (Álvarez, 2008): plantear las tareas de evaluación como tareas de aprendizaje, involucrar a las y los estudiantes en la evaluación y ofrecer los resultados de la evaluación a modo de feedback.

En nuestro caso, algunas de las actividades (EP5 y EP6) son evaluadas por otro grupo de alumnos/as, o bien por otro grupo y además por la profesora (EP10), o bien realizando una autoevaluación así como coevaluación de sus compañeros/as del trabajo aportado al grupo durante el proyecto (EP9). En general, el sistema de evaluación es el siguiente: las actividades del proyecto califican un $50 \%$ y el resto otro $50 \%$, de los cuales, los controles individuales de contenidos mínimos suponen un $40 \%$. Para aprobar los controles es necesario obtener un $60 \%$ de la nota, tanto en la parte teórica como en la parte práctica. Se realizan dos en cada asignatura. Si no se supera el primero habrá una repesca de esa parte en el segundo control. Los otros entregables de la asignatura valen un $10 \%$ en total. Para evaluar la calidad de los entregables se facilitan rúbricas que tienen en consideración tanto aspectos formales como de aplicación técnica de los conocimientos y procedimientos de contabilidad requeridos en cada caso.

\subsubsection{Los cinco ingredientes para el aprendizaje cooperativo}

Las y los estudiantes cooperan para aprender porque deben presentar entregables que son grupales, porque la exposición oral que haga una persona supondrá parte de la calificación de todo el grupo, porque en algunos casos corregirán el trabajo de otros grupos y, finalmente, porque se verán obligados/as a valorar las aportaciones de los otros miembros de su grupo (y serán igualmente valorados/as). Garantizamos que el alumnado ha adquirido los conocimientos o competencias mínimas mediante la realización de los exámenes individuales. Si no obtienen un $60 \%$ de la nota de cada control (ni en la repesca), irán al examen final con la parte que les quede por liberar.

Por lo tanto, ésta es la forma en que se incorporan al proyecto los cinco ingredientes para el aprendizaje cooperativo:

1. Exigibilidad individual: además de aportar en el trabajo del grupo, cada estudiante debe preparar su aportación a los entregables grupales, debe corregir los entregables de otros/as y debe superar los exámenes individuales. 
2. Interacción cara a cara: la interacción positiva se dará en todas las sesiones que trabajan en grupo, ya sea en clase o en horario no presencial.

3. Habilidades interpersonales y de trabajo en grupo: las habilidades de responsabilidad, cumplimiento de las responsabilidades, negociación a la hora de tomar decisiones, etc. serán necesarias para poder cumplir con las tareas en los plazos especificados.

4. Interdependencia positiva: deben asegurarse de que aportan al trabajo del grupo, ya que sus compañeros/as les evaluarán por su aportación, y deben asegurarse que la persona que haga la exposición oral del trabajo (cualquier miembro al azar) tenga un adecuado conocimiento de lo que han hecho entre todos/as.

5. Reflexión del grupo: la reflexión se inicia la 2. ${ }^{\mathrm{a}}$ semana, al poner en común las posibles disfuncionalidades del trabajo en equipo y firmar un contrato de normas propio. Además, cada vez que realizan la entrega de partes del trabajo del proyecto deben reflexionar sobre cómo han planificado y realizado el trabajo, si todos los miembros del grupo han colaborado y valorar el trabajo en equipo de cada uno de ellos, así como proponer acciones de mejora.

\section{Implantación en el aula, seguimiento y resultados del proyecto}

La primera implantación de este proyecto se inició en septiembre de 2013 y finalizó en mayo de 2014. Las aulas disponen de mobiliario fijo (en filas de 3 o 4 asientos cada una) que no facilita especialmente el trabajo en equipo, pero lo utilizamos así. En el diseño inicial se detalló qué temas se iban a relacionar específicamente con el proyecto, pero realmente todos los temas se impartieron en base a metodologías cooperativas, siendo necesaria la preparación de algún tipo de entregable, de asignatura o de proyecto, para cada uno de ellos.

\subsection{Puesta en marcha}

Una vez que el material básico para la asignatura se había puesto a disposición del alumnado en el Centro de Publicaciones de la UPV/EHU (incluida la Guía Estudiante con el listado de entregables y sus fechas aproximadas de entrega, que también estaba en la plataforma virtual eGela, tipo Moodle), en la primera sesión de la asignatura se presentó la metodología y sistema de evaluación general que iba a tener lugar a lo largo de las dos asignaturas (el resumen de la presentación se facilita en una hoja fotocopiada y también se dispone en Moodle). Por otra parte, se les indicó que debían ir definiendo voluntariamente los grupos fijos de 3 personas en los que iban a desarrollar el proyecto. El primer día se preguntó en general sobre los conocimientos previos. Como en años anteriores, se observó que había una parte menor del alumnado que había cursado 
alguna optativa de Economía en el Bachillerato y que podía tener cierta idea de elaborar Balances de Situación, pero el punto de partida de la asignatura es considerar que inicialmente no saben nada de contabilidad.

En la segunda sesión, a partir de las lecturas facilitadas en el taller ERAGIN, se realizó una reflexión sobre posibles disfuncionalidades de los grupos (incluyendo una puesta en común general), y se preparó un entregable con la reflexión de cada grupo de proyecto y sus normas firmadas. La metodología general consistió en que cada miembro del grupo preparaba una parte del tema y, después de explicar su parte al resto de miembros del grupo, completaban colaborativamente la actividad correspondiente. Así, se empezó a trabajar en el tema 1 con la preparación de su entregable que consistía en completar un mapa conceptual sobre la Contabilidad: qué es, para qué sirve, principales personas y entidades usuarias, tipos de decisión para la que necesitan información, etc. El «planteamiento del proyecto" (explicación por parte de la profesora de la pregunta motriz, así como formulación de las preguntas guía, planificación de objetivos por parte de cada grupo, etc.) se dejó para la segunda semana, una vez que ya tenían unos conocimientos mínimos sobre contabilidad.

La participación general fue de 3 personas por grupo. Por lo tanto, se formaron entre 7 y 12 equipos en cada grupo de matrícula, si bien algunos equipos se fueron mermando por abandonos, quedando en 2 personas por grupo. En un grupo el éxito fue total: todas las personas que comenzaron el proyecto lo finalizaron. En cambio, en los otros dos grupos, cuatro personas no finalizaron el proyecto, ya que lo abandonaron en su fase inicial o en la final, según la situación personal de acumulación de asignaturas del curso anterior, motivos de salud, etc. Pero, en general, se trabajó de forma homogénea mediante el desarrollo del proyecto a lo largo de toda la asignatura.

\subsection{Seguimiento y resultados de la primera implementación}

El ambiente de trabajo en general fue bueno en todos los grupos. También es cierto que algunas personas mostraron ciertas reticencias sobre las metodologías empleadas, sobre todo, al inicio. Parte del alumnado prefería la clase magistral, porque los conceptos se explican desde el principio y es el sistema al que se habían acostumbrado. Aunque se aceptara el hecho de que la profesora no iba a explicar el tema de forma tradicional, sino que lo preparan en grupo, algunos/ as alumnos/as se quejaban del «estrés» que produce estar contando el tiempo (5 minutos de lectura individual, 10 minutos para explicarlo en grupo, etc.), o bien de que el nivel de asimilación de las distintas partes puede ser diferente, según le haya tocado preparárselo o no, los distintos ritmos de aprendizaje, etc.

En todo caso, valoramos positivamente la realización de actividades de co-evaluación en las que un grupo de estudiantes, a partir de la documentación que incluye la teoría y los criterios de corrección correspondientes, califica la activi- 
dad realizada por otro grupo y, en su caso, discute y reclama supervisión de la profesora respecto de la calificación de su actividad propia, que le ha sido otorgada también por otro grupo. Las principales dificultades en la implementación se relacionan con la falta de asistencia ocasional (especialmente si se trata de finalizar una actividad en grupo comenzada la sesión anterior) o con la entrada en clase una vez iniciada la sesión, etc., unida a la falta de trabajo no presencial individual (estudio pre- y post-sesión, realización de supuestos prácticos, etc.). Al final del primer cuatrimestre, observamos que, aunque el alumnado/a en general ya se había acostumbrado al nuevo sistema de trabajo, todavía quedaban reticencias sobre el sistema (algunos recomendarían volver a la enseñanza tradicional, que se explique más en clase, etc.), reconociendo que es una materia difícil para su preparación de forma autónoma. El programa se implementó sin apenas modificaciones respecto al plan diseñado inicialmente, tanto en las actividades presenciales como en las no presenciales, incluidas las actividades de evaluación.

Las actividades de evaluación de las dos asignaturas se implementaron tal como estaban previstas, relacionadas con las competencias para las que se diseñaron, en la semana en la que se había especificado al inicio del curso, y en las condiciones de retroalimentación que se habían previsto: por un lado, las de coevaluación se califican por otro grupo en el aula y en caso de reclamación, el grupo de origen tiene ocasión de discutir la nota en ese mismo momento con la profesora (normalmente para subirla, porque el grupo calificador es muy exigente), por otro lado, las que corrige la profesora se enseñan calificadas en clase en la sesión posterior o en un plazo breve de tiempo y en otros casos, los menos, acuden a tutorías a revisar la actividad.

En cuanto a los resultados, en primer lugar podemos decir que fueron positivos tanto en IC como en CF, ya que la mayoría del alumnado que terminó el proyecto (se mantuvo en evaluación continua hasta el final) aprobó en la convocatoria ordinaria y con buenas calificaciones. Pero a la hora de analizar las tasas de éxito y rendimiento en ambas asignaturas, es necesario tener en cuenta las características de los grupos docentes existentes. La mayor parte del alumnado del grupo 01 (de la mañana en castellano) es de primera matrícula, ya que quienes repiten asignatura o curso se matriculan en el grupo 16 (de tarde). En el momento de matricularse eligen el turno (de mañana o tarde) en el que quieren estudiar por orden de nota en el expediente académico, de modo que son los/as mejores alumnos/as quienes eligen primero. Así, el grupo 16 está formado por alumnado que trabaja en actividades remuneradas fuera de la universidad o por personas que repiten asignatura o curso, de forma que algunas o no asisten a clase y no se presentan, o cuando asisten lo compaginan con asignaturas de primer curso, lo que aumenta su carga de trabajo global y hace que su rendimiento en nuestra asignatura sea menor que la del grupo 01. En el grupo 31, de euskera, no hay posibilidad de elección de turno, por lo que se mezcla alumnado de 
primera matrícula con personas repetidoras, teniendo parte de los condicionantes del grupo 16.

\subsubsection{Resultados de la implantación del proyecto en la asignatura Introducción a la Contabilidad}

Al término del proyecto, cada estudiante debe ser capaz de obtener los resultados de aprendizaje relacionados con las competencias específicas de la asignatura, que se han obtenido mediante la realización de tareas que incluyen los entregables tanto de asignatura como de proyecto. Pensamos que quien ha realizado los entregables y otras tareas no evaluables, tanto en el aula como de forma no presencial, con la finalidad de lograr los resultados de aprendizaje prefijados, debería obtener buenos resultados globales al término de cada asignatura. En todo caso, los resultados del primer curso de implantación en Introducción a la Contabilidad fueron los siguientes:

Tabla 5

Comparación del rendimiento académico en el primer año de implementación del proyecto respecto del curso anterior en la asignatura Introducción a la Contabilidad

\begin{tabular}{l|cc|cc|cc}
\hline \multirow{2}{*}{\multicolumn{1}{c|}{ Tasas }} & \multicolumn{2}{|c|}{ Grupo 01 } & \multicolumn{2}{c|}{ Grupo 16 } & \multicolumn{2}{c}{ Grupo 31 } \\
\cline { 2 - 7 } & $2012 / 13$ & $2013 / 14$ & $2012 / 13$ & $2013 / 14$ & $2012 / 13$ & $2013 / 14$ \\
\hline Evaluación =Present./matr. & $81 \%$ & $92 \%$ & $70 \%$ & $67 \%$ & $78 \%$ & $73 \%$ \\
Éxito =Aprob./present. & $89 \%$ & $89 \%$ & $68 \%$ & $75 \%$ & $82 \%$ & $68 \%$ \\
Rendimiento =Aprob./matr. & $72 \%$ & $82 \%$ & $48 \%$ & $50 \%$ & $63 \%$ & $50 \%$ \\
\hline
\end{tabular}

Fuente: Elaboración propia.

El grupo 01 de la mañana, que parecía que era el que más reticencias presentaba ante la nueva metodología, obtuvo mejores resultados, con un porcentaje de presentación del $92 \%$ sobre matrícula, y una tasa de aprobado sobre presentación del $89 \%$. En el grupo 16 se presentó un $67 \%$ del alumnado y aprobó el $75 \%$. Sigue siendo un buen resultado, pero es cierto que es una diferencia de 15 puntos que no atribuimos a la metodología utilizada, sino a las características de este grupo antes mencionadas. Y si comparamos estos resultados con los resultados del curso anterior, vemos que mejoran los resultados en algunos indicadores y en otros se mantienen. En el grupo 01 aumentó el porcentaje de presentados 11 puntos y aunque la tasa de éxito se mantiene, lógicamente aumenta la tasa de rendimiento, pasando del $72 \%$ al $82 \%$. En el grupo 16 , sin embargo, el aumento de la tasa de rendimiento se debe al incremento del porcentaje de superación sobre matrícula, ya que el porcentaje de presenta- 
ción desciende 3 puntos. Los resultados del grupo 31 de euskera empeoraron en todos los indicadores, pero creemos que se debió fundamentalmente a que en el curso anterior se observaba una mejor capacidad inicial y motivación para el estudio en el grupo de personas matriculadas (que incluso obtuvieron algunas Matrículas de Honor).

Para hacernos una idea de lo que opinaba el alumnado sobre la metodología utilizada, analizamos los resultados de la encuesta facilitada por el programa ERAGIN que cumplimentaron los y las estudiantes de la primera implantación del proyecto al término de la asignatura del primer cuatrimestre. Las valoraciones negativas se concentraron en dos aspectos: en cuanto a la organización de grupos y sobre todo del tiempo de trabajo en el aula; y en cuanto a poner en relación el trabajo autónomo grupal respecto de las explicaciones de la profesora en un método tradicional, decantándose por esta última opción. Así, obtuvimos las siguientes valoraciones negativas:

- Se pierde mucho tiempo al establecer los grupos.

- Cronometrar cada paso no lo encuentro lógico.

- Los tiempos de lectura y comprensión son muy breves.

- Trabajar todos juntos en clase bien, pero estaría bien explicar la lectura en clase.

- Más explicaciones en clase y que no se base todo en resolver y explicar entre nosotros.

- Aprendemos trabajando pero se echan en falta más explicaciones.

- Hay demasiados entregables.

— No logro entender la materia con esta metodología.

Con la pregunta de si cambiarían algo, obtuvimos las siguientes propuestas:

- Dar más tiempo para no tener tanta presión al realizar los ejercicios.

- Realizar entregables de aula en casa para hacerlos con más tiempo.

- Más casos prácticos.

- Hay muchas prácticas que cuentan poco. Preferiría hacer menos prácticas pero más difíciles y que contaran más.

- La actividad del poster parecía de la ESO.

- Preferiría hacer los entregables solo/a.

- Que el/a profesor/a recalque las cosas importantes después de poner en común.

- Que la profesora explique, que no nos mande leerlo a nosotros.

- Mantener prácticas grupales pero la teoría con ayuda de la profesora.

- No cambiaría nada porque se estimula la participación y se dan todos los aspectos para aprender y entender la materia dada. 
Pero también encontramos valoraciones positivas con los siguientes argumentos:

- Muy práctica. Hay trabajo individual y nos obliga a trabajar en equipo.

- Al principio me parecía un mal método, pero ahora me doy cuenta que el método nos ayuda a asentar más los conocimientos porque lo tengo que trabajar yo.

— La metodología facilita el aprendizaje.

- El material facilita el aprendizaje y la disponibilidad en tutorías.

- Trabajamos en grupo y las prácticas hacen que entendamos los temas por nosotros mismos.

- Clase más amena al realizar más tareas.

- Te obliga a trabajar en clase.

- Metodología buena para el desarrollo de competencias.

- Se va aprendiendo poco a poco en clase y consiguiendo puntos.

- Me ha venido bien a la larga para «sacarme las castañas del fuego».

- Con las prácticas y el trabajo en equipo es más fácil comprender la materia.

Por lo tanto, aunque había quien opinaba que cronometrar cada paso resulta agobiante, que no logran entender la materia con esta metodología y que estaría bien explicar la lectura en clase (que no se base todo en resolver y explicar entre ellos/as), en general, reconocen que aprenden trabajando.

\subsubsection{Resultados de la implantación del proyecto en la asignatura Contabilidad Financiera}

La asignatura CF es una continuación de la asignatura Introducción a la Contabilidad, por lo que resulta fundamental haber comprendido y adquirido las habilidades básicas en la primera asignatura (elaboración del Balance de Situación y Cuenta de Pérdidas y Ganancias, registro de las operaciones contables tanto en el Libro Diario como en el Mayor, etc.) para poder afrontar la segunda con garantías. En ocasiones, esto no se cumple y la segunda les resulta más difícil, de ahí que los resultados tradicionalmente hayan sido peores, porque suele haber más abandonos y peores tasas de rendimiento, de modo que existe mayor cantidad de personas que repiten la asignatura (cursando además otras del curso siguiente) que en el primer cuatrimestre.

Los resultados del curso 2013/2014 fueron positivos en el grupo 01. La mayoría de quienes aprobaron obtuvieron calificaciones de Notable y Sobresaliente, e incluso hubo tres Matrículas de Honor. El grupo 16 de tarde, que como ya hemos mencionado elige este turno porque trabaja por las mañanas, porque está cursando asignaturas de primero por las mañanas, o simplemente porque tiene peor expediente académico, es lógico que obtenga peores resultados. 
Tabla 6

Comparación del rendimiento académico en el primer año de implementación del proyecto respecto del curso anterior en la asignatura Contabilidad Financiera

\begin{tabular}{l|cc|cc|cc}
\hline \multirow{2}{*}{\multicolumn{1}{c|}{ Tasas }} & \multicolumn{2}{|c|}{ Grupo 01 } & \multicolumn{2}{c|}{ Grupo 16 } & \multicolumn{2}{c}{ Grupo 31 } \\
\cline { 2 - 7 } & $2012 / 13$ & $2013 / 14$ & $2012 / 13$ & $2013 / 14$ & $2012 / 13$ & $2013 / 14$ \\
\hline Evaluación =Present./matr. & $71 \%$ & $60 \%$ & $39 \%$ & $44 \%$ & $57 \%$ & $41 \%$ \\
Éxito =Aprob./present. & $89 \%$ & $74 \%$ & $80 \%$ & $45 \%$ & $74 \%$ & $71 \%$ \\
Rendimiento =Aprob./matr. & $63 \%$ & $53 \%$ & $31 \%$ & $20 \%$ & $43 \%$ & $29 \%$ \\
\hline
\end{tabular}

Fuente: Elaboración propia.

En todo caso, los resultados de los tres grupos fueron peores que en el 2012/2013 en todos los indicadores, excepto un ligero aumento en la tasa de evaluación en el grupo 16. En el grupo 01 y 16 disminuye la tasa de rendimiento debido a la disminución de la de éxito (en el grupo 01 pasa del $89 \%$ al $74 \%$, y en el 16 del $80 \%$ al $45 \%$ ), mientras que la tasa de evaluación se mantiene en el grupo 01 e incluso aumenta en el grupo 16. En cambio, en el grupo 31 la disminución de la tasa de rendimiento se debe a la disminución de la tasa de evaluación (16 puntos) mientras que la tasa de éxito disminuye de forma poco significativa.

En definitiva, los resultados cambiaron, pero no podemos determinar en qué medida influyó el cambio de metodología puesto que en cursos anteriores, aunque no utilizábamos una metodología tan activa, sí se hacía evaluación continua ( 2 trabajos en grupo y 2 controles individuales) tal como explicamos en trabajos anteriores (Vázquez y Saitua, 2007). Además, en el curso 2012-2013, habíamos incorporado como novedad el hecho de que no fuera necesario acudir al examen final si aprobaban en el $2 .^{\circ}$ control, lo que ya había promovido la mejora de resultados respecto de cursos anteriores.

\subsubsection{Discusión de los primeros resultados y sus implicaciones para el futuro}

\section{a) Aspectos que salieron bien en la implementación}

La metodología implementada en clase mediante el desarrollo del proyecto ayuda a ver el interés profesional que tiene la materia que se imparte, si bien anteriormente ya realizaban la búsqueda de las cuentas anuales en el Registro Mercantil como parte de un trabajo en grupo y, por lo tanto, esta actividad no era tan novedosa. Por otra parte, tenemos claro que las asignaturas de contabilidad no son centrales en la formación del grado en Relaciones Laborales y Recursos Humanos, por lo que la motivación del alumnado puede ser menor al pen- 
sar que su desempeño profesional no va a ir por ese camino. Pero en el pasado, cuando se ha encuestado a nuestro alumnado sobre las distintas asignaturas, sí responden que estas asignaturas pueden ser de utilidad, ya que, por ejemplo, al trabajar en una asesoría que abarque distintas áreas de actividad (laboral fiscalcontable, etc.) puede ser que terminen llevando a cabo tareas relacionadas con nuestras asignaturas. En todo caso, el alumnado de grupos de tarde que ya trabaja en alguna empresa (aunque sea en otro tipo de tareas como soldador, etc.) suele reconocer en mayor medida el interés por conocer la información financiera para poder evaluar mejor la evolución de la situación de la empresa en la que están interesados/as.

En el primer cuatrimestre el alumnado se acostumbró a trabajar con la nueva metodología y en el segundo hubo menos problemas. A pesar de algunas reticencias, finalmente reconocieron muchas ventajas de su implementación. Reconocieron que trabajando en equipo es más fácil la comprensión de un determinado tema. Se consigue que la clase sea más amena porque están siempre ocupados/as y los conocimientos se van asentando porque ellos/as aportan de forma individual desde el primer momento y con su aportación al grupo se van haciendo dueńos/as de su aprendizaje.

\section{b) Aspectos para reformar}

Los aspectos considerados negativos por parte del alumnado nos llevaron a las siguientes reflexiones:

- Control de tiempos en tareas presenciales. Al principio también a las profesoras nos supuso una novedad tener que estar controlando los minutos. Con el tiempo hemos aprendido a flexibilizarlo y adaptarlo a las necesidades de los grupos, si bien el límite está determinado por el nivel de objetivos que se pretenda lograr. Es decir, que si se espera al grupo más retardado se corre el riesgo de disminuir el nivel de cumplimiento del programa. En todo caso, una vez que el alumnado superó la experiencia del $1 .{ }^{\text {er }}$ cuatrimestre (incluida la valoración final), en la asignatura del $2 .^{\circ}$ cuatrimestre observamos que el alumnado ya está acostumbrado desde el principio y las posibles reticencias que puedan surgir en este sentido se resuelven más fácilmente. De hecho las quejas sobre este aspecto disminuyeron.

- Trabajo autónomo-individual vs explicación previa de la profesora. Consideramos normal que existieran reticencias sobre la forma de enfrentarse a los temas por primera vez, puesto que, en general, a lo largo de su vida académica anterior habían estado acostumbrados/as a un sistema más tradicional, en el que el profesor/a era la pieza fundamental. Sin embargo, en general, van siendo conscientes de que el proceso de aprendizaje es, sobre todo, responsabilidad del alumnado. En ocasiones, aún dudan de 
la metodología porque requiere del compromiso del grupo y en el $2 .^{\circ}$ cuatrimestre se han dado cuenta de las diferencias que hay entre ellos/as y han surgido problemas. Pero no puede responsabilizarse tanto a la metodología, sino a la falta de compromiso individual.

- Las calificaciones grupales diluyen los esfuerzos individuales. Como en todo sistema que incluya calificaciones grupales, puede ocurrir que un/a estudiante con un examen de Sobresaliente vea minorada su nota final a causa de las notas medias de entregables de grupo. También se da, y más frecuentemente, el caso contrario de alumnado que en el examen no pasa de un 6, pero que llega al Notable gracias a las tareas de grupo. Creemos que es un aspecto de «injusticia valorativa» que no se puede olvidar, que debemos saber transmitir al alumnado desde el principio que esto puede ocurrir, y en algunos casos en que consideremos necesario, aplicar cierta «flexibilidad» (más subjetiva en base a la valoración global que hacemos del/a estudiante en cuestión) a la hora de poner la nota final. Debemos dejar claro que el grupo puede ayudar o perjudicar en la nota final. En todo caso, es necesario trabajar en el grupo y sacar adelante las tareas, tanto por compromiso hacia los compañeros/as, como por el propio rendimiento individual.

\section{c) Conclusiones y valoración global de la primera implantación}

Para las profesoras la experiencia resultó enriquecedora en el sentido de que hemos comprobado que el alumnado puede aprender de forma alternativa a la tradicional, que se basa en la lección magistral y en la resolución de ejercicios prácticos. La formulación de una pregunta motriz como eje del proyecto ayuda a visualizar la utilidad de cada una de las asignaturas en la vida real y, por lo tanto, entendemos que fomenta la motivación del alumnado para su implicación en el esfuerzo individual y grupal que se exige para poder superar dichas asignaturas. Por otro lado, el hecho de realizar entregables que califican en horario presencial nos evita tener que "pasar lista" para controlar la asistencia. Aunque todavía hay parte del alumnado que no asiste a algunas sesiones, y otros/as que estando matriculados/as, no asisten en todo el cuatrimestre (repetidores/as que se están dedicando a otras asignaturas y dejan ésta para más adelante) y/o que habiéndose encuadrado en el proyecto terminan abandonando. Sin embargo, la asistencia de los inscritos/as en los grupos de trabajo es generalizada. En cuanto al nivel de adquisición de competencias por parte del alumnado, entendemos que fue igual o mayor a cursos anteriores debido a la mayor intensidad de puesta en común de los conocimientos (en grupo pequeño para realizar el entregable, en grupo grande cuando la profesora resume o comenta algo, en grupo pequeño al calificar el trabajo de otros, y después al comprobar la calificación recibida...), habiéndose desarrollado el $100 \%$ del temario. 


\subsection{Evolución posterior de los resultados académicos, implicaciones y nuevas tareas}

En el curso 2013/2014 pusimos en práctica la metodología basada en proyecto y la dinámica posterior ha sido similar durante todo el periodo analizado, esto es, continuamos realizando las mismas actividades, el sistema de evaluación también es el mismo, las profesoras que impartimos la materia somos las mismas y la coordinación en la docencia es total.

Sin embargo, a partir de la experiencia de la primera implantación, en el curso 2014/2015 se dieron algunos cambios que se mantienen en la actualidad. Buscando garantizar que individualmente se consiguen las competencias y visto que parte del alumnado asistía a clase, pero en los controles únicamente respondía las preguntas teóricas, o incluso solo venía a firmar, decidimos incorporar un nuevo requisito de nota mínima para seguir en la evaluación continua (3/10 puntos en los controles individuales), con el objetivo de que asumiesen que el trabajo de clase (entregables que califican en grupo) solo vale si se le añade un mínimo trabajo individual no presencial. En todo caso, tanto en el grupo 16 como en el grupo 31, se va acumulando cierta cantidad de personas que repiten asignatura o curso, de modo que normalmente empeoran la tasa de rendimiento del grupo, ya sea en forma de disminución de la tasa de evaluación (porque en ocasiones dejan la asignatura para examinarse en otro curso posterior) o de la de éxito (porque cuando se examinan lo hacen en peores condiciones de preparación que las personas que acuden de forma continuada y que cumplen con los requisitos de la evaluación continua).

\subsubsection{Análisis de los resultados de un periodo de cinco cursos académicos}

Si comparamos los datos de 2013/2014 (de primera implantación de la metodología de ABPy), con los del curso 2016/2017, observamos que la tasa de rendimiento ha disminuido en todos los grupos excepto en CF en el grupo 01 y 16, pero si analizamos el periodo total desde el curso 2012/2013 sí se cumple el descenso global en todos los indicadores. En todo caso, la mayoría de aumentos

\section{Gráfico 1}

Evolución de las tasas de Rendimiento de IC y CF en cada grupo desde 2012/2013 hasta el 2016/2017
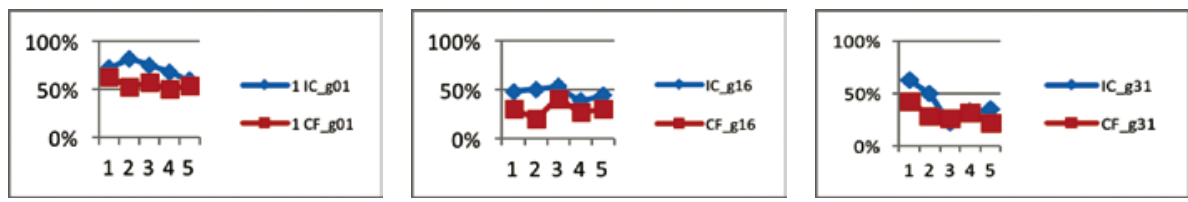

Fuente: Elaboración propia. 
o disminuciones en las tasas de rendimiento que se dan en una asignatura se dan también de forma similar en la otra asignatura del mismo curso académico, ya que la mayoría del alumnado que forma el grupo de matrícula es el mismo.

En Introducción a la Contabilidad, el grupo 01 que partía de la tasa más alta de rendimiento en 2012/2013 (72\%) y que mejoró con la implantación del ABPy, ha ido reduciendo progresivamente hasta llegar en 2016/2017 a una tasa del $59 \%$. Las razones han sido la disminución de la tasa de evaluación (8 puntos, del $81 \%$ al $73 \%$ ), así como la de éxito (quien aprueba tras presentarse) que se ha reducido en la misma medida (del 89\% al 81\%), pero manteniéndose con porcentajes elevados de éxito. En el grupo 16, la tasa de rendimiento (48\%), que también mejoró con la implantación del $\mathrm{ABPy}$, se ha reducido 4 puntos (del $48 \%$ al 44\%) y el motivo también es la disminución de la tasa de evaluación en el último curso, si bien en los cursos intermedios se había incrementado. Al igual que en el grupo 01, la tasa de éxito ha aumentado (del 68\% al 83\%) remontando una tasa muy reducida que se produjo en el curso 2015/2016. En este sentido, destaca la elevada tasa en el grupo $16(83 \%)$ que supera la tasa del grupo 01, tradicionalmente más elevada. Por último, en el grupo 31 de euskera, que partía de una tasa de rendimiento del $63 \%$ y que empeoró con la implantación de la metodología ABPy, se reduce hasta el 35\% en 2016/2017, si bien se

Tabla 7

Evolución de las tasas de Evaluación, Éxito y Rendimiento desde 2012/2013 hasta el 2016/2017

\begin{tabular}{|c|c|c|c|c|c|c|c|c|c|c|c|}
\hline \multirow{2}{*}{ Tasas } & \multirow{2}{*}{ Grupo } & \multicolumn{5}{|c|}{ IC } & \multicolumn{5}{|c|}{$\mathrm{CF}$} \\
\hline & & $2012 / 13$ & $2013 / 14$ & $2014 / 15$ & $2015 / 16$ & $2016 / 17$ & $2012 / 13$ & $2013 / 14$ & $2014 / 15$ & $2015 / 16$ & $2016 / 17$ \\
\hline \multirow{3}{*}{$\begin{array}{c}\text { Evaluación } \\
= \\
\text { Present./matr. }\end{array}$} & 01 & $81 \%$ & $92 \%$ & $84 \%$ & $84 \%$ & $73 \%$ & $71 \%$ & $60 \%$ & $65 \%$ & $66 \%$ & $70 \%$ \\
\hline & 16 & $70 \%$ & $67 \%$ & $71 \%$ & $72 \%$ & $53 \%$ & $39 \%$ & $44 \%$ & $52 \%$ & $48 \%$ & $46 \%$ \\
\hline & 31 & $78 \%$ & $73 \%$ & $48 \%$ & $67 \%$ & $62 \%$ & $57 \%$ & $41 \%$ & $27 \%$ & $32 \%$ & $39 \%$ \\
\hline \multirow{3}{*}{$\begin{array}{c}\text { Éxito } \\
\quad= \\
\text { Aprob./eval }\end{array}$} & 01 & $89 \%$ & $89 \%$ & $89 \%$ & $81 \%$ & $81 \%$ & $89 \%$ & $74 \%$ & $88 \%$ & $76 \%$ & $76 \%$ \\
\hline & 16 & $68 \%$ & $75 \%$ & $76 \%$ & $53 \%$ & $83 \%$ & $80 \%$ & $45 \%$ & $78 \%$ & $57 \%$ & $65 \%$ \\
\hline & 31 & $82 \%$ & $68 \%$ & $46 \%$ & $50 \%$ & $57 \%$ & $74 \%$ & $71 \%$ & $100 \%$ & $100 \%$ & $56 \%$ \\
\hline \multirow{3}{*}{$\begin{array}{c}\text { Rendimiento } \\
= \\
\text { Aprob./matr. }\end{array}$} & 01 & $72 \%$ & $82 \%$ & $75 \%$ & $68 \%$ & $59 \%$ & $63 \%$ & $53 \%$ & $57 \%$ & $50 \%$ & $54 \%$ \\
\hline & 16 & $48 \%$ & $50 \%$ & $53 \%$ & $38 \%$ & $44 \%$ & $31 \%$ & $20 \%$ & $41 \%$ & $27 \%$ & $30 \%$ \\
\hline & 31 & $63 \%$ & $50 \%$ & $22 \%$ & $33 \%$ & $35 \%$ & $43 \%$ & $29 \%$ & $27 \%$ & $32 \%$ & $22 \%$ \\
\hline
\end{tabular}

Fuente: Elaboración propia. 
observa que su evolución es más irregular, quizá porque la menor cantidad de alumnado matriculado hace que cualquier variación de personas que se presentan, aprueban, etc. supongan mayores porcentajes en las tasas. Al igual que en los grupos de castellano, uno de los motivos de esta reducción ha sido el descenso de la tasa de evaluación (16 puntos, del $78 \%$ al 62\%), aunque en este caso también se ha producido una disminución importante de la tasa de éxito del $82 \%$ al $57 \%$.

Con todo, en el último curso en todos los grupos se ha reducido la tasa de evaluación, debido a que parte del alumnado ha abandonado la evaluación continua y luego no se ha presentado al examen final (convocatoria ordinaria). Esta podría ser la razón de que la tasa de éxito mejore con respecto al curso anterior (2015/2016), en el sentido de que las personas que han continuado en evaluación continua son las personas interesadas y motivadas, de forma que superan la asignatura en un porcentaje más elevado, si bien no llegan a las tasas que existían en el momento de la primera implantación de la metodología ABPy en 2014/2015. Pero, en todo caso, entendemos que las razones se dan en el propio colectivo de alumnado. En la medida en que otras asignaturas implementan sistemas que exigen un trabajo más continuado, hay parte del alumnado que «gestiona» su currículum eligiendo a qué asignaturas se presenta y decide no hacerlo a contabilidad. A ello contribuye la mayor libertad y facilidad para la renuncia de las convocatorias que la que había, por ejemplo, en las antiguas titulaciones de licenciatura y diplomatura. O puede ser que acceda a la universidad ya con un nivel de preparación y cultura del esfuerzo menor que las generaciones anteriores.

En CF, el grupo 01, que también partía de la tasa más alta de rendimiento (63\%) y que empeoró con la implementación del ABPy en 2013/2014, ha ido reduciendo progresivamente hasta llegar, en 2016/2017, a una tasa del $54 \%$. En este caso, la razón principal ha sido la disminución de la tasa de éxito (13 puntos, del $89 \%$ al $76 \%$ ), en tanto que la de evaluación (70\%) vuelve a ser similar a la de 2012/2013. En el grupo 16 la tasa de rendimiento (30\%) se redujo al $20 \%$ con la implementación ABPy y en el último curso vuelve a ser similar a la de 2012/2013, si bien en los cursos intermedios se había incrementado. Por último, en el grupo 31 de euskera la tasa de rendimiento se reduce 21 puntos (del $43 \%$ al $22 \%$ ). Al igual que en los grupos de castellano, uno de los motivos de esta reducción ha sido el descenso de la tasa de evaluación (18 puntos, del $57 \%$ al $39 \%$ ), aunque, en este caso, también se ha producido una disminución de la de éxito del $74 \%$ al $56 \%$.

\subsubsection{Implicaciones para el futuro y nuevas tareas}

Basándonos en la experiencia de más de 20 años de docencia en las asignaturas, podemos decir que el alumnado que asiste a clase y lleva la materia al día, 
que está dentro de este proceso y tiene interés por aprender, en general, aprueba la asignatura. Pero dados los resultados académicos posteriores a la implantación del proyecto ERAGIN, observamos que, a pesar de nuestro esfuerzo, los mejores resultados ante una metodología novedosa no son indefinidos. Independientemente de que cada grupo docente es diferente, resulta difícil mantener la atención y motivación de todas las personas matriculadas, si bien en nuestras asignaturas deben estar realizando actividades constantemente. Por lo tanto, creemos necesario continuar realizando acciones que busquen concienciar al alumnado para que se mantenga en la evaluación continua y realice todas las actividades del proyecto hasta el final. Así, para el curso 2017/2018 plantearemos dos nuevas tareas.

Tabla 8

Nuevas actividades a realizar en Introducción a la Contabilidad y Contabilidad Financiera

\begin{tabular}{|c|c|c|}
\hline \multirow{2}{*}{ Contexto } & \multicolumn{1}{|c|}{ Visita a empresa } & Búsqueda de artículos sobre temas contables \\
\cline { 2 - 3 } & \multicolumn{1}{|c|}{$\begin{array}{c}\text { «Introducción a la Contabilidad» } \\
\text { El alumnado del Grado en Relaciones Laborales y Recursos Humanos tiene como } \\
\text { Problema } \\
\text { detectado } \\
\text { (aplicación del Derecho del Trabajo a la determinación del tipo de contrato, las re- } \\
\text { muneraciones que corresponden, las altas y bajas en la Seguridad Social, etc.) así } \\
\text { como el asesoramiento y gestión de los recursos humanos (aplicación de los conoci- } \\
\text { mientos de Psicología del Trabajo y Organización de Empresa a la selección de per- } \\
\text { sonal, acogida, formación y desarrollo, etc.) y no tanto la llevanza de la contabilidad, } \\
\text { de forma que algunas personas la toman como una asignatura alejada de su posible } \\
\text { perfil profesional futuro, a lo que se ańade que otras la consideran difícil (por tra- } \\
\text { tar con números). Esto hace que parte del alumnado afronte la asignatura con cierta } \\
\text { desmotivación y predisposición al fracaso. }\end{array}$} \\
\hline $\begin{array}{c}\text { Objetivo } \\
\text { general } \\
\text { perseguido }\end{array}$ & $\begin{array}{l}\text { Concienciar al alumnado de la importancia de la contabilidad y los datos que se } \\
\text { obtienen de ella (resultado del ejercicio, saldo de tesorería, impuestos a pagar, divi- } \\
\text { dendos a repartir, etc.), tanto para poder realizar valoraciones de la situación econó- } \\
\text { mico-financiera de una empresa a la hora de tomar decisiones del tipo de contratar } \\
\text { personal o no, aumentar salarios o no, etc., como de la relación que tiene la activi- } \\
\text { dad de asesoría laboral con la de asesoría contable y fiscal en la realidad práctica de } \\
\text { las empresas. }\end{array}$ \\
\hline
\end{tabular}


EXPERIENCIA EN METODOLOGÍAS ACTIVAS: PROYECTO PARA LAS ASIGNATURAS INTRODUCCIÓN A LA CONTABILIDAD

y Contabilidad Financiera en el grado de Relaciones Laborales y Recursos Humanos de la UPV/EHU

\begin{tabular}{|c|c|c|}
\hline \multirow{2}{*}{ Contexto } & Visita a empresa & Búsqueda de artículos sobre temas contables \\
\hline & «Introducción a la Contabilidad» & «Contabilidad Financiera» \\
\hline $\begin{array}{l}\text { Objetivo } \\
\text { concreto } \\
\text { perseguido }\end{array}$ & $\begin{array}{l}\text { Facilitar el aprendizaje del alum- } \\
\text { nado mediante una actividad grupal, de } \\
\text { encuentro con profesionales del sector } \\
\text { del asesoramiento laboral-contable o de } \\
\text { la gestión de recursos humanos, en el } \\
\text { que podrá formular preguntas, para fo- } \\
\text { mentar su motivación en el aprendizaje } \\
\text { de la asignatura, al sentirse protagonista } \\
\text { del mismo y tener valor en la califica- } \\
\text { ción final. }\end{array}$ & $\begin{array}{l}\text { Facilitar el aprendizaje del alumnado } \\
\text { mediante una actividad on-line en grupo, } \\
\text { de búsqueda de información externa a los } \\
\text { materiales y fuentes facilitados por las pro- } \\
\text { fesoras, para fomentar su motivación en } \\
\text { el aprendizaje de la asignatura, al sentirse } \\
\text { protagonista del mismo y tener valor en la } \\
\text { calificación final. }\end{array}$ \\
\hline $\begin{array}{l}\text { Intervención } \\
\text { educativa }\end{array}$ & $\begin{array}{l}\text { Una vez que se ha estudiado el ci- } \\
\text { clo contable y su normalización básica } \\
\text { (temas 1-6), se realizará una visita vo- } \\
\text { luntaria a empresa (en grupo reducido, } \\
\text { seleccionando de entre las personas in- } \\
\text { teresadas, según la nota que hayan ob- } \\
\text { tenido en el primer control individual) } \\
\text { que les dará la oportunidad de ver (no } \\
\text { en un puesto de trabajo concreto, sino } \\
\text { en una sala de reuniones), cómo se } \\
\text { crearon las empresas, cuál fue la for- } \\
\text { mación académica y experiencia profe- } \\
\text { sional de quien las creó, cuáles son los } \\
\text { principales servicios que ofrecen (aseso- } \\
\text { ramiento y/o gestión laboral, contable, } \\
\text { etc.), cómo han ido evolucionando a lo } \\
\text { largo del tiempo, cuáles son algunos de } \\
\text { los programas informáticos que utilizan } \\
\text { para ello, los problemas que suelen sur- } \\
\text { gir en el día a día, etc. } \\
\text { La actividad se realizará en grupos } \\
\text { de } 5 \text {-10 personas (según restricciones } \\
\text { impuestas por la empresa a visitar) y } \\
\text { será voluntaria. Cada estudiante relle- } \\
\text { nará un cuestionario que tendrá una va- } \\
\text { loración máxima ańadida de } 0,5 \text { puntos } \\
\text { en la calificación final, una vez que se } \\
\text { haya superado la asignatura. }\end{array}$ & $\begin{array}{l}\text { En la asignatura que trata las normas } \\
\text { de reconocimiento y valoración de te- } \\
\text { mas específicos (adquisición de inmovili- } \\
\text { zado, inversiones financieras, provisiones } \\
\text { de pasivo, distribución del resultado, etc.), } \\
\text { las profesoras diseńarán una base de da- } \\
\text { tos, que aparecerá en la plataforma virtual } \\
\text { "eGela" (Moodle) en cada uno de los te- } \\
\text { mas elegidos. Se trata de que el alumnado } \\
\text { busque y cite un recurso (prensa, artículo } \\
\text { académico, capítulo de libro, reseńas de } \\
\text { libro, enlaces web, etc.) relativo a alguna } \\
\text { operación/tema del programa, insertando } \\
\text { en la base de datos, además de los datos } \\
\text { correspondientes a la cita (título, auto- } \\
\text { ría, etc.), el archivo en pdf, la imagen de } \\
\text { la reseña en cuestión, o la URL de la pá- } \\
\text { gina web cuando sea posible. Además, en } \\
\text { un campo de texto libre, deberán comen- } \\
\text { tar brevemente las normas de valoración y } \\
\text { registro relacionadas con la operación es- } \\
\text { tudiada, así como incorporar la referencia } \\
\text { bibliográfica correspondiente. } \\
\text { La actividad se realizará en grupos de } \\
2 \text { personas y será obligatoria. Tendrá una } \\
\text { valoración máxima de } 1 \text { punto en la cali- } \\
\text { ficación final de la asignatura. Cada grupo } \\
\text { elaborará la tarea en un único tema, lo que } \\
\text { supone una labor previa de la docente para } \\
\text { distribuir un tema a cada grupo/equipo de } \\
\text { trabajo. }\end{array}$ \\
\hline
\end{tabular}




\begin{tabular}{|c|c|c|}
\hline \multirow{2}{*}{ Contexto } & Visita a empresa & Búsqueda de artículos sobre temas contables \\
\hline & «Introducción a la Contabilidad» & «Contabilidad Financiera» \\
\hline $\begin{array}{c}\text { Resultados } \\
\text { esperados }\end{array}$ & $\begin{array}{l}\text { Durante la visita el alumnado re- } \\
\text { llenará un cuestionario que se le facili- } \\
\text { tará antes de la visita para poder recoger } \\
\text { las informaciones que les explican en la } \\
\text { misma. Se espera que reconozcan la im- } \\
\text { portancia de la gestión de contabilidad } \\
\text { en la empresa ya sea interna o externa- } \\
\text { lizada, ver los programas informáticos } \\
\text { que usan para llevar la contabilidad, sa- } \\
\text { ber que se utiliza la información conta- } \\
\text { ble para asesorar en la negociación de } \\
\text { convenios colectivos, que la empresa } \\
\text { puede pedir certificado de anteceden- } \\
\text { tes por tema de prevención de resposa- } \\
\text { bilidad empresarial, reconocer la nece- } \\
\text { sidad de seguir aprendiendo a lo largo } \\
\text { de toda la vida profesional, etc. En de- } \\
\text { finitiva, que conozcan experiencias rea- } \\
\text { les relacionadas con lo que estudian y } \\
\text { lo que se van a encontrar en el futuro } \\
\text { profesional. }\end{array}$ & $\begin{array}{l}\text { Se espera motivar al alumnado me- } \\
\text { diante la utilización de herramientas cola- } \\
\text { borativas (Moodle, en este caso), que faci- } \\
\text { litan realizar la actividad on-line y también } \\
\text { su corrección on-line por parte de la pro- } \\
\text { fesora. Una vez que se ha realizado la en- } \\
\text { trega y se ha evaluado, el alumnado podrá } \\
\text { visualizar el trabajo de sus compañeros/as. } \\
\text { Por una parte se pretende que mejore } \\
\text { el desarrollo de competencias específicas } \\
\text { (asimilación de conceptos, visibilización } \\
\text { de su relación con la realidad empresarial, } \\
\text { etc.) de la asignatura. Además, se desarro- } \\
\text { llan competencias transversales como el } \\
\text { trabajo en equipo o el tratamiento de la } \\
\text { información, este último imprescindible } \\
\text { para realizar un TFG de calidad. }\end{array}$ \\
\hline
\end{tabular}

Fuente: Elaboración propia.

Al finalizar se les pasará una encuesta en la que se les preguntará por su nivel de valoración de la actividad (1-Nada, 2-Poco, 3-Regular, 4-Bastante, 5-Mucho): si les ha gustado, si creen que han aprendido algo útil para la asignatura, para la titulación, para su futuro profesional, y con un campo de texto para preguntas sobre si habían realizado alguna actividad similar en otra asignatura (en cuál), qué es lo que más les ha llamado la atención (sorprendido), gustado, resultado difícil, o no han entendido, etc.

\section{Conclusiones y reflexiones finales}

El objetivo de este artículo consistía en presentar la evolución de los resultados de la implementación del Aprendizaje Basado en Proyecto (ABPy) para las asignaturas de Introducción a la Contabilidad y Contabilidad Financiera en el grado de Relaciones Laborales y Recursos Humanos, analizando las tasas de rendimiento académico en un periodo de 5 cursos. Nuestra opinión como docentes es que el alumnado puede aprender de forma alternativa a la tradicional basada 
en la lección magistral y en la resolución de ejercicios prácticos. La formulación de una pregunta motriz como eje del proyecto ayuda a visualizar la utilidad de cada una de las asignaturas en la vida real y, por lo tanto, entendemos que fomenta la motivación del alumnado para su implicación en el esfuerzo individual y grupal que se exige para poder superar dichas asignaturas.

Los resultados de la primera implantación de la metodología ABPy fueron positivos, ya que la mayoría de quienes terminaron el proyecto aprobaron las asignaturas con buenas calificaciones. Sin embargo, en varios de los grupos se obtuvieron peores resultados si atendemos a las tasas de rendimiento académico. Posteriormente, desde el curso 2013/2014, de primera implantación de la metodología de ABPy, hasta el curso 2016/2017, la tasa de rendimiento ha disminuido en casi todos los grupos, tanto en Introducción a la Contabilidad como en Contabilidad Financiera, y este descenso se confirma si tomamos como partida para el análisis el curso 2012/2013, anterior a la primera implantación de la metodología ABPy. Este descenso en el rendimiento se debe fundamentalmente a una reducción en la tasa de evaluación. Teniendo en cuenta que durante todos los años analizados las profesoras que hemos impartido las asignaturas hemos sido las mismas, que hemos aplicado la misma metodología en todos los grupos y que llevamos a cabo la planificación, desarrollo y evaluación diaria de todas las tareas académicas del alumnado perfectamente coordinadas, entendemos que el descenso en la tasa de evaluación debe atribuirse al colectivo del alumnado. Además, como es lógico, la mayoría de aumentos o disminuciones en las tasas de rendimiento que se dan en una asignatura se dan también de forma similar en la otra asignatura del mismo curso académico ya que la mayoría del alumnado que forma el grupo de matrícula es el mismo. Por lo tanto, entendemos que la clave reside en que cada vez una mayor parte del alumnado decide abandonar la evaluación continua (así como la final) de las asignaturas de contabilidad, ya sea porque otras asignaturas también implementan sistemas que exigen un trabajo más continuado y en un momento dado interpretan que la carga es excesiva, o bien porque acceden a la universidad ya con un nivel de preparación y cultura del esfuerzo menor que las generaciones precedentes. Como limitaciones, resaltamos el hecho de que los datos que se utilizan para realizar los informes sobre el desarrollo docente del centro y que han servido como base para este análisis corresponden a la convocatoria ordinaria, si bien es verdad que hay quien aprueba posteriormente en la convocatoria extraordinaria, resultando las tasa finales ligeramente superiores.

Finalmente, entendemos que este artículo supone una contribución en varios sentidos. Por un lado, da cuenta de los esfuerzos de la UPV/EHU por actualizar los métodos docentes en un marco de adaptación a la nueva realidad sociocultural actual y, en concreto, de la realizada en estas asignaturas que pueden servir de ejemplo tanto para otras asignaturas en los grados relacionados con las relaciones laborales y recursos humanos, como para otros grados en los que se 
imparten las asignaturas de contabilidad analizadas. Así, creemos que el planteamiento del proceso de enseñanza-aprendizaje formulado en forma de proyecto mejora la adquisición de algunas de las competencias que más demandan las empresas hoy en día, como son la gestión de la información, la adaptación a nuevas formas de crear conocimiento y la capacidad de colaboración en equipo.

A futuro, el profesorado deberá seguir formándose y aplicar innovaciones docentes con el objetivo de mantener la motivación por el aprendizaje por parte del alumnado. Sin embargo, estamos convencidas de que, por mucho esfuerzo pedagógico que realice el profesorado, es el alumnado quien finalmente determina con sus capacidades iniciales y, después, con su asistencia, actitud y esfuerzo continuado en el tiempo, el mayor o menor éxito en su proceso de aprendizaje y, a la postre, su nivel de éxito en la superación de las materias correspondientes. Futuros estudios podrán continuar analizando la implantación de esta y otras metodologías activas en otras asignaturas y grados, de otras universidades y contextos geográficos, para avanzar en el conocimiento sobre cómo afectan las decisiones en el planteamiento educativo sobre el rendimiento académico y la satisfacción global obtenidos, de forma que se pueda continuar tomando las decisiones de gestión universitaria más adecuadas para obtener la mejor preparación posible de las personas que van a egresar en las distintas titulaciones.

\section{Bibliografía}

Aliane, Nourdine (2006): «Una experiencia de aprendizaje basado en proyecto en una asignatura de robótica». Jornadas de Innovación Universitaria, 3. Obtenido de http:// abacus.universidadeuropea.es/handle/11268/3426 (último acceso 14/07/2017).

Alvárez, Ibis (2008): «Evaluación del aprendizaje: unas mirada rertrospectiva y prospectiva desde la divulgación científica», Revista Electrónica de Investigación psicoeducativa - Electronic Journal of Research in Educational Psychology, 14 (1), 235-272. E-ISSN: 1696-2095, Universidad de Almería. Obtenido de http://www.redalyc.org/ pdf/2931/293121940013.pdf (último acceso 14/07/2017).

Carrasco, Amalia; Donoso, José Antonio; Duarte, Teresa; Hernández, José Julián; López, Rosario y Núñez, Carmen (2009): «Aprendizaje Basado en Proyectos versus Aprendizaje Basado en Actividades: una experiencia en la en la elaboración y análisis de estados financieros", ASEPUC, VI Jornadas de Docencia y Contabilidad. https:// www.researchgate.net/publication/255623535_APRENDIZAJE_BASADO_EN_ PROYECTOS_VERSUS_APRENDIZAJE_BASADO_EN_ACTIVIDADES_ UNA_EXPERIENCIA_EN_LA_ELABORACION_Y_ANALISIS_DE_LOS_ ESTADOS_FINANCIEROS (último acceso 14/07/2017).

Casasola Balsells, María Araceli; Pérez Chamorro, Vicente Antonio; García ÁlvaREZ DE PereA, Juan (2012): «Aprendizaje basado en proyectos y trabajo en equipo: innovando en la docencia de la asignatura Sistemas contables informatizados». Revista de Innovación Docente UPO INNOVA, 1, 107-122. https://www.upo.es/revistas/ index.php/upoinnova/article/view/89 (último acceso 14/07/2017) 
Cullen, John; Richardson, Sue y O’Brien, Rona (2004): «Exploring the teaching potential of empirically-based case studies». Accounting Education 13 (2): 251-266 http://www.tandfonline.com/doi/abs/10.1080/09639280420001676648 (último acceso 14/07/2017).

Domínguez Alfonso, Rocío (2009): «La sociedad del conocimiento y los nuevos retos educativos».Etic@net. Publicación en línea. Granada (España). 7 (8), 1-19, ISSN: 1695-324X. Obtenido de http://www.ugr.es/ -sevimeco/revistaeticanet/index.html (último acceso acceso 07/06/2017).

Fernández, Idoia y Alkorta, Itziar (2014): «Capítulo 2, El aprendizaje activo como reto: razones visibles e invisibles de una política de desarrollo docente en la UPV/EHU», en Guisasola, Jenaro y Garmendia, Mikel (Ed.): Aprendizaje basado en problemas, proyectos y casos: diseño e implementación de experiencias en la universidad, 31-87. Servicio Editorial de la Universidad del País Vasco (UPV/EHU) Disponible en https://addi. ehu.es/handle/10810/12368 (último acceso 05/07/2017).

Gessa Perera, Ana (2011): "La coevaluación como metodología complementaria de la evaluación del aprendizaje: análisis y reflexión en las aulas universitarias», Revista de Educación 344, 749-764. ISSN 0034-8082. http://rabida.uhu.es/dspace/bitstream/ handle/10272/10762/La_coevaluacion_como_metodologia.pdf?sequence=2 (último acceso14/07/2017).

Guisasola, Jenaro y Garmendia, Mikel (2014): «Capítulo 1, El programa ERAGIN de formación en metodologías activas de la UPV/EHU», en Guisasola, Jenaro y Garmendia, Mikel (Ed.): Aprendizaje basado en problemas, proyectos y casos: diseño e implementación de experiencias en la universidad, pp. 13-30. Servicio Editorial de la Universidad del País Vasco (UPV/EHU) Disponible en https://addi.ehu.es/ handle/10810/12368 (último acceso 05/07/2017).

Saitua, Ainhoa y VÁzquez, Isabel (2014): «Elaboración de la contabilidad de una empresa comercial». IKD baliabideak 8. Universidad del País Vasco (UPV/EHU). http:// cvb.ehu.es/ikd-baliabideak/saitua-08-2014.htm [Fecha de acceso 14/07/2017]

Solomon, Gwen (2003): «Project-based learning: a primer» http://pennstate.swsd. wikispaces.net/file/view/pbl-primer-www_techlearning_com.pdf (último acceso 14/07/2017).

UPV/EHU (Universidad del País Vasco) (2011): Convocatoria ERAGIN. Servicio de Asesoramiento Educativo. Vicerrectorado de Estudios de grado e Innovación. Obtenido de http://www.ehu.eus/es/web/sae-helaz/eragin-deialdia (últimoacceso 07/06/2017).

VÁzquez, Isabel y Saitua, Ainhoa (2007a): «El desarrollo de competencias. Propuesta para la formación en el área contable del nuevo Grado en Relaciones Laborales y Recursos Humanos de la UPV/EHU». Lan Harremanak. Revista de Relaciones Laborales. Los estudios y las prácticas profesionales en Relaciones Laborales y Recursos Humanos. 17(2), 139-162. Servicio Editorial Universidad del País Vasco/EHU, Bilbao, ISSN 1575-7048 http://www.ehu.eus/ojs/index.php/Lan_Harremanak/article/view/3078 (último acceso 14/07/2017).

VÁzquez, Isabel y SaItua IrIbar, Ainhoa (2007b): «Iniciativas para la introducción del Sistema Europeo de Créditos: la experiencia de la asignatura 'Introducción a la Contabilidad' en la Escuela Universitaria de Relaciones Laborales de la Universidad del País Vasco/EHU». Revista Trabajo, Universidad de Huelva, 19, 115-136, Huelva, ISSN 1136-3819 http://www.uhu.es/publicaciones/ojs/index.php/trabajo/article/ view/93 (último acceso 14/07/2017). 